\title{
Characterization of free volume during vulcanization of styrene butadiene rubber by means of positron annihilation lifetime spectroscopy and dynamic mechanical test
}

\author{
A. J. Marzocca, ${ }^{1}$ S. Cerveny, ${ }^{1}$ W. Salgueiro, ${ }^{2}$ A. Somoza, ${ }^{2}$ and L. Gonzalez ${ }^{3}$ \\ ${ }^{1}$ Universidad de Buenos Aires, Facultad de Ciencias Exactas y Naturales, Departamento Física, Laboratorio de Propiertades Mecanicas \\ de Polimeros y Materiales Compuestos, Ciudad Universitaria, Pabellon 1, Buenos Aires (1428), Argentina \\ ${ }^{2}$ Universidad Nacional del Centro de la Provincia de Buenos Aires, Instituto de Fisica de Materiales de Tandil, \\ Pinto 399, Tandil (7000), Argentina \\ and Comisión de Investigaciones Científicas de la Provincia de Buenos Aires, Buenos Aires, Argentina \\ ${ }^{3}$ Instituto de Ciencia y Tecnología de Polímeros (CSIC), Juan de la Cierva 3, 28006, Madrid, Spain \\ (Received 13 December 2000; revised manuscript received 23 May 2001; published 15 January 2002)
}

\begin{abstract}
An experimental investigation was performed to study the effect on the free volume of the advance of the cross-linking reaction in a copolymer of styrene butadiene rubber by sulfur vulcanization. The dynamic modulus and loss tangent were evaluated over samples cured for different times at $433 \mathrm{~K}$ by dynamic mechanical tests over a range of frequencies between 5 and $80 \mathrm{~Hz}$ at temperatures between 200 and $300 \mathrm{~K}$. Using the William-Landel-Ferry relationship, master curves were obtained at a reference temperature of $298 \mathrm{~K}$ and the coefficients $c_{1}^{0}$ and $c_{2}^{0}$ were evaluated. From these parameters the dependence of the free volume on the cure time is obtained. Positron annihilation lifetime spectroscopy was also used to estimate the size and number density of free volume sites in the material. The spectra were analyzed in terms of continuous distributions of free volume size. The results suggest an increase of the lower free volume size when cross linking takes place. Both techniques give similar results for the dependence of free volume on the time of cure of the polymer.
\end{abstract}

DOI: 10.1103/PhysRevE.65.021801

PACS number(s): 61.41.+e, 36.20.-r, 78.70.Bj

\section{INTRODUCTION}

It is known that, on increasing the cure level in sulfur vulcanized elastomers, the mechanical dynamic response, estimated by means of the variation of the dynamic moduli with frequency, shows a shift of the transition zone to lower frequencies [1-3]. In the case of natural rubber vulcanizates this shift is due mainly to the presence of side reactions and secondarily to the increase in cross-link density [4-6]. Direct consequences of this are the hindrance of chain mobility and a broadening of the mechanical relaxation curves $[7,8]$. These facts are associated with a broadened distribution of the free volume of the system [8].

The experimental determination of the free volume in polymeric systems is of great importance not only from the theoretical point of view but also for practical applications. In practice the experimental determination of free volume is not an easy task.

Since the experimental determination of the free volume is not simple, several theoretical frameworks have been used to get an indirect estimation of the free volume from experimental results. In the case of dynamic mechanical measurements, the William-Landel-Ferry (WLF) relationship [9] is one of the most popular methods based on the timetemperature principle to evaluate the free volume fraction. It can be estimated by means of the shift factor $a_{T}$ evaluated from analysis of the dependence of the storage modulus on frequency and temperature by applying the WLF relationship. The shift factor is expressed by

$$
\log _{10} a_{T}=\frac{-c_{1}^{0}\left(T-T_{0}\right)}{\left(c_{2}^{0}+T-T_{0}\right)},
$$

where $c_{1}^{0}$ and $c_{2}^{0}$ are constants evaluated at the reference temperature $T_{0}$ and $T$ is the temperature in kelvins.

The fractional free volume $f$ is given by $f=v_{f} / v$ where $v_{f}$ and $v$ are the free and total volumes, respectively. The total volume is the sum of the free volume and an occupied volume $v_{0}$ which includes not only the volume of the molecules as represented by their van der Waals radii but also the volume associated with vibrational motions [9]. The shift factor $a_{T}$ is related to the fractional free volume by [9]

$$
\log _{10} a_{T}=\frac{B}{2.303}\left(\frac{1}{f}-\frac{1}{f_{0}}\right)
$$

where $f_{0}$ is the fractional free volume evaluated at the reference temperature $T_{0}$ and $B$ is an empirical constant near unity.

A few novel techniques have been applied to estimate the free volume in polymers: wide-angle x ray diffraction, photoisomerization of photochromic probe molecules [10], and positron annihilation lifetime spectroscopy (PALS). Specifically, PALS has been used to evaluate the free volume and pore size in materials as diverse as silica gels and zeolites and in the study of biological macromolecules [11-14].

A positron injected into a polymeric material can pick up an electron from the material and form a neutral particle called positronium $(\mathrm{Ps})$ which occurs in two states, parapositronium ( $\mathrm{p}-\mathrm{Ps}$, antiparallel positron-electron spins) and ortho-positronium (o-Ps, parallel spins). These two states are formed according to the ratio 1:3 between p-Ps and o-Ps. The probability of Ps formation depends on the physical and chemical properties of the materials. Precisely, from the annihilation process of the Ps, it is possible to obtain information regarding the free volume distribution. In fact, the lifetime of p-Ps (0.123 ns in vacuum) is too short to be influenced by the volume factors. As the characteristic life- 
time of o-Ps is long enough (140 ns in vacuum), it is possible that the positron in the o-Ps state can annihilate with an electron other than its bound partner (and of opposite spin), following a process commonly called pick-off annihilation (for more details see [12]).

An empirical approximation allows us to describe the correlation between free volume size and the long-lived lifetime component corresponding to o-Ps. In the approximation proposed by Tao [15] and by Eldrup et al. [16], it is assumed that o-Ps resides in a simple potential well, such as a spherical well of radius $R$ having an infinite potential barrier. Then, the following semiempirical expression is usually used:

$$
\tau_{\mathrm{o}-\mathrm{Ps}}=0.5\left[1-\frac{R}{(R+\delta R)}+\frac{1}{2 \pi} \sin \left(\frac{2 \pi R}{(R+\delta R)}\right)\right]^{-1},
$$

where $\delta R$ is the thickness $(0.166 \mathrm{~nm})$ of the electron layer that constitutes the wall of the hole and can overlap with the o-Ps wave function. In some cases, the long-lived component results in a lifetime distribution; as a consequence a free volume distribution is obtained $[11,17]$

The number of free volume holes in the material is represented by the relative intensity $I_{\mathrm{o}-\mathrm{Ps}}$ corresponding to the o-Ps component $\tau_{\text {o-Ps }}$. The free volume fraction $f$ in polymers is assumed to be proportional to the density of holes and to the average volume of each hole. Then the following semiempirical equation is used:

$$
f=A v_{h} I_{\mathrm{o}-\mathrm{Ps}},
$$

where $v_{h}$ is the volume of the hole and $A$ is a constant

In this paper, the behavior of the free volume was investigated during the vulcanization of styrene butadiene rubber (SBR) at $433 \mathrm{~K}$. By means of dynamic mechanical tests, the changes in the loss tangent and storage modulus at different temperatures and frequencies with different cure conditions are presented. Using the method of reduced variables, the master curves of the dynamic moduli against frequency were obtained at a reference temperature. Then the values of $\log _{10}\left(a_{T}\right)$ as a function of temperature were evaluated and consequently the parameters of the WLF relationship. With this information it is possible to correlate the fractional free volumes obtained using PALS with those of the free volume theory and confirm its validity.

\section{EXPERIMENTAL TECHNIQUES}

The material used in the present study was SBR1502. This is a copolymer of styrene and butadiene that contains $23.5 \%$ bound styrene, i.e., a molecular proportion of one styrene for every six or seven butadienes. The chemical structure of butadiene in the SBR copolymer consist of 55\% trans-1.4, 9.5\% cis-1.4, and 12\% 1,2-butadiene. The molecular weight $M_{n}=176000 \mathrm{~g} / \mathrm{mol}$ was obtained by gel permeation chromatography.

A compound of unfilled SBR was prepared according to the formulation given in Table I in a laboratory mill.

Samples prepared as sheets of $150 \times 150 \times 2 \mathrm{~mm}^{3}$ were cured for 12.6, 15.1, 18.7, and $31.2 \mathrm{~min}$ at $433 \mathrm{~K}$. According to rheometer curves [18], the cure level $(\gamma)$ corresponding to
TABLE I. Compound formulation.

\begin{tabular}{lc}
\hline \hline Ingredient & Per hundred rubber \\
\hline SBR1502 & 100.0 \\
Zinc oxide & 5.0 \\
Stearic acid & 2.0 \\
Antioxidant & 1.2 \\
TBBS $^{\mathrm{a}}$ & 1.2 \\
Sulfur & 1.8 \\
\hline \hline
\end{tabular}

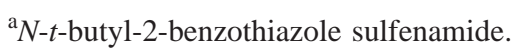

each time is $0.5,0.75,0.9$, and 1.0, respectively. Samples were quenched in ice and water after being removed from the mold. For measurements of the dynamic properties, strip samples of $15 \times 5 \times 2 \mathrm{~mm}^{3}$ were cut with a die.

The storage modulus $\left(E^{\prime}\right)$, loss modulus $\left(E^{\prime \prime}\right)$, and loss tangent $(\tan \delta)$ were measured using a Metravib viscoelasticimeter in the frequency range between 5 and $80 \mathrm{~Hz}$ and the temperature range between 200 and $300 \mathrm{~K}$ using a temperature ramp of $5 \mathrm{~K} / \mathrm{min}$. The Metravib was used in tension compression mode with a fixed displacement of $2 \mathrm{~mm}$ thus ensuring linear viscoelastic behavior.

A fast-fast timing coincidence system was used as lifetime spectrometer for the PALS measurements. A time resolution (full width at half maximum) of $255 \mathrm{ps}$ was obtained using the code RESOLUTION [19]. The positron lifetime spectra were recorded at room temperature with a counting rate of about 100 counts/s and a total number of $(2-3) \times 10^{6}$ coincidence counts using a $20 \mu \mathrm{Ci}$ source of ${ }^{22} \mathrm{Na}$ deposited on a Kapton foil (7.5 $\mu \mathrm{m}$ thick). The source was placed between two identical disk-shaped samples of $2 \mathrm{~mm}$ thick and $15 \mathrm{~mm}$ diameter obtained from the same sheets used for the dynamic mechanical test. As is usual in polymers the PALS spectra were deconvoluted into three components, two discrete and one giving the o-Ps lifetime distribution, using the LT program [20] with an adequate source correction.

\section{RESULTS AND DISCUSSION}

From PALS measurements and by using Eq. (3), the average volume of the hole $v_{h}=4 \pi R^{3} / 3$ can be calculated in spherical approximation. The values of the normalized intensities $I_{\mathrm{o}-\mathrm{Ps}}$ and the mean free volume $v_{h}$ for all the analyzed samples are given in Table II. In this table the value $f / A$ is given according to Eq. (4).

By analyzing the PALS spectra, the distribution of inverse lifetimes $\xi\left(1 / \tau_{\mathrm{o}-\mathrm{Ps}}\right)$ can be estimated. This distribution is related to the radius distribution $f(R)$ as [21]

TABLE II. Dependence of positron parameters on the cure time.

\begin{tabular}{cccc}
\hline \hline Cure time $(\min )$ & $\gamma$ & $v_{h}\left(\AA^{3}\right)$ & $I_{\mathrm{o}-\mathrm{Ps}}(\%)$ \\
\hline 0 & 0 & $122.6 \pm 1.2$ & $24.4 \pm 0.8$ \\
12.6 & 0.5 & $118.2 \pm 0.8$ & $23.3 \pm 0.9$ \\
15.1 & 0.75 & $118.7 \pm 1.0$ & $21.9 \pm 1.1$ \\
18.7 & 0.90 & $119.7 \pm 0.9$ & $24.4 \pm 1.0$ \\
31.2 & 1.00 & $120.2 \pm 0.6$ & $26.8 \pm 0.8$ \\
\hline \hline
\end{tabular}




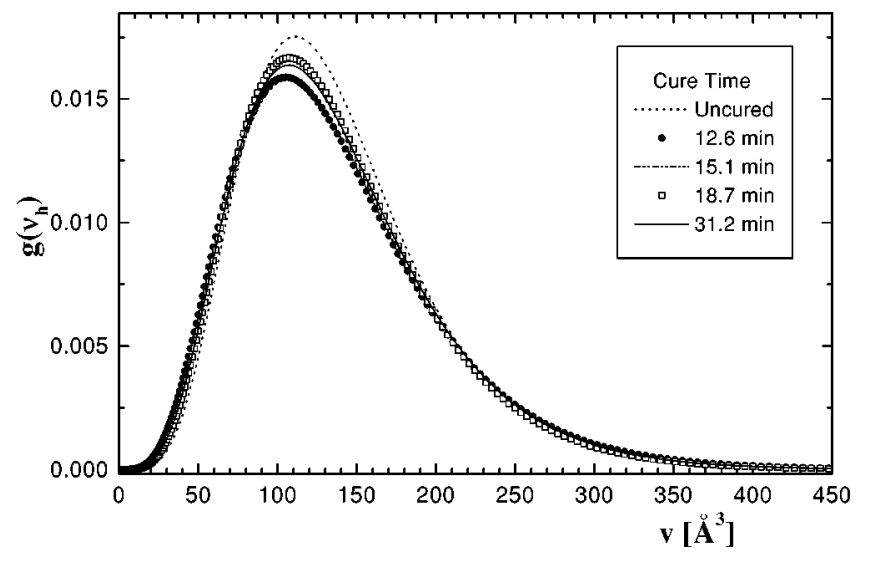

FIG. 1. Free volume distributions $g\left(v_{h}\right)$, for uncured SBR and SBR cured for different times.

$$
f(R)=2 \delta R \cos \left(\frac{2 \pi R}{R+\delta R}-1\right) \frac{\xi\left(1 / \tau_{\mathrm{o}-\mathrm{Ps}}\right)}{(R+\delta R)^{2}}
$$

Then it is easy to obtain the free volume distribution as $g\left(v_{h}\right)=f(R) / 4 \pi R^{2}$. The fraction of positrons annihilating in cavities with volumes between $v_{h}$ and $v_{h}+d v_{h}$ is $g\left(v_{h}\right) d v_{h}$ [11].

The dependence of $g\left(v_{h}\right)$ on the time of cure in the samples of SBR cured at $433 \mathrm{~K}$ is given in Fig. 1. All the distributions consist of one skewed peak and extend from about $20 \AA^{3}$ to $450 \AA^{3}$. Some features must be mentioned in analyzing these plots. The behavior of the most probable free volume size with the cure time is shown in Fig. 2. From this figure we are able to analyze the evolution of free volume during the cure of the material.

We can observe that the free volume decreases significantly at the beginning of the cure. This fact implies a lower freedom of movement of the macromolecular segments in the cured state compared to the uncured state. This phenomenon is consistent with the macroscopic changes that are produced at the glass transition temperature in the cured and uncured states [22]. Thus it can be concluded that the presence of cross links decreases the volume of holes in the sample. Once a minimum value is obtained the free volume

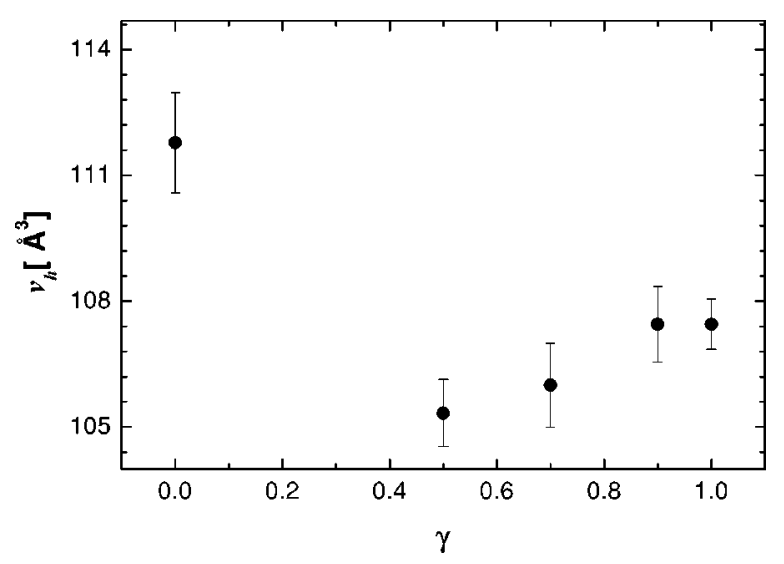

FIG. 2. Average values of the free volume obtained from Fig. 1 at different cure levels. increases until we reach the maximum cure level. So we conclude that the free volume passes through a minimum value during the cure of material. A similar trend was observed by Venditti et al. [23] in an epoxy/amine system. In that work, it was proposed that an antiplasticization process occured during the cure of the material. The unreacted material behaves as an antiplasticizer.

In our samples the cure system is composed of sulfur, an accelerator (TBBS), and an activator (zinc oxide). It is well known that in sulfur vulcanization only part of the combined sulfur goes to form cross links between chains [24]. Also, a variety of chemical reactions take place during the curing process and these lead to a variety of structures [24,25]. For example, during the vulcanization the network contains dangling accelerator-derived groups of the type $-S_{x}$-Ac where Ac is the radical of an accelerator molecule and $S_{x}$ represents a cross link. We identify these structures with antiplasticizers since they fulfill the requirements of these structures [23].

These structures are consumed during the curing of the material, forming the cross-linking network. In this way, the antiplasticizer molecules fill the polymer free volume at the beginning of the curing and they are consumed during the process, increasing the free volume.

As can be understood from Figs. 1 and 2, the other interesting fact is that there is a broadening of the free volume distribution when comparing the uncured sample with the cured ones. This confirms the early analysis of Mason [8] who inferred that the broadening of the mechanical relaxation curves of natural rubber with increase in the degree of cross linking was due to a broadened distribution of the free volume in the polymer.

The master curves of the storage modulus $E^{\prime}$, loss modulus $E^{\prime \prime}$, and loss tangent $\tan \delta$ for the cured samples of SBR at a reference temperature $T_{0}=298 \mathrm{~K}$ are shown in Figs. 3(a), 3(b), and 3(c). The parameters of the WLF relationship, Eq. (1), used to built the master curves are given in Table III for each sample measured.

According to the literature, the following relationships connect the WLF parameters $c_{1}^{0}$ and $c_{2}^{0}$ with the free volume fraction $f[9]$ :

$$
\begin{gathered}
c_{1}^{0}=\frac{B}{2.303} f_{0}, \\
c_{2}^{0}=\frac{f_{0}}{\alpha_{f}}, \\
\alpha_{f}=\frac{B}{2.303} c_{1}^{0} c_{2}^{0},
\end{gathered}
$$

where $\alpha_{f}$ is the thermal expansion of the free volume relative to the total volume. For the sake of simplicity, $B$ has usually been set arbitrarily equal to unity. Then from Eq. (6) the fraction of free volume $f_{0}$ at the reference temperature $T_{0}$ can be calculated and the corresponding values are given in Table III. The fraction of free volume is nearly constant between $\gamma=0.5$ and 0.75 and increases at high cure levels. This fact might be associated not only with the creation of new 


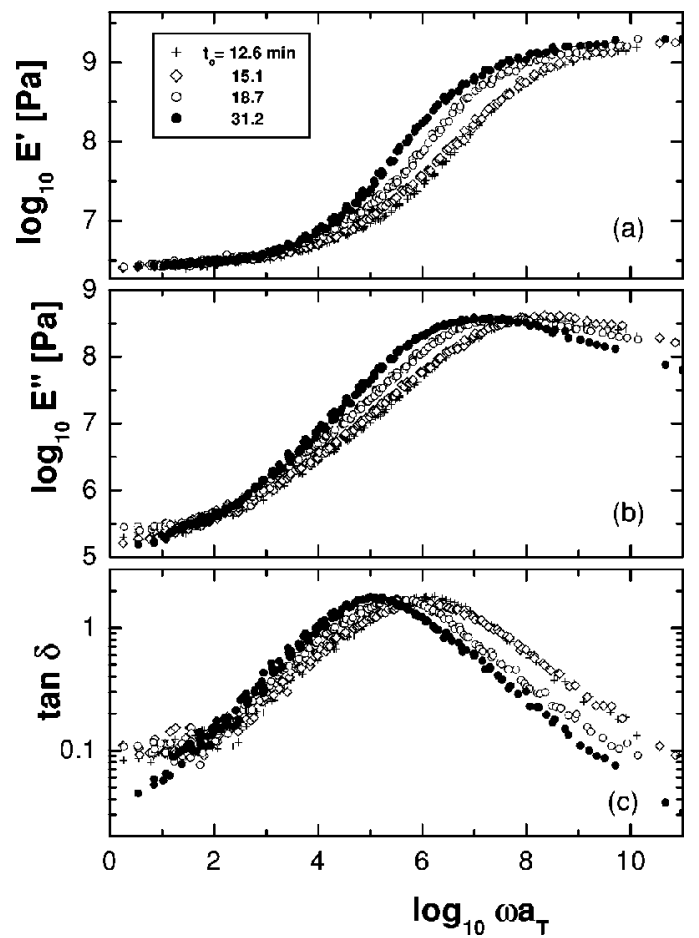

FIG. 3. Master curves from WLF model of SBR vulcanizates at a reference temperature $T_{0}$ of $298 \mathrm{~K}$.

cross links during curing but also with the side reactions produced during sulfur vulcanization [5]. Srithawatpong et al. [26] recently reported measurements of free volume hole sizes in cured cis-polyisoprene, high vinyl polybutadiene, and their 50:50 mixture. They observed that on cross linking both the average hole size and the free volume fraction decrease. But some remarks can be made regarding this research. First, the different cross-link densities in the samples were obtained by changing the concentration of the cross-linking agent and not the curing time as in our investigation. Second, in the case of the results presented for cispolyisoprene and high vinyl polybutadiene, only one level of cure was examined with respect to the uncured sample. Finally, in the case of the blend a minimum value of the free volume was reported when the cross linking increased, as in the present report.

By using Eq. (8), the parameter $\alpha_{f} / B$ was estimated and is also given in Table III. The values are close to those reported in the literature for the same elastomer [9] and seem to increase with increasing degree of cure.

Banduzuch et al. [27] recently reported a study using information from PALS and viscosity data of some uncured

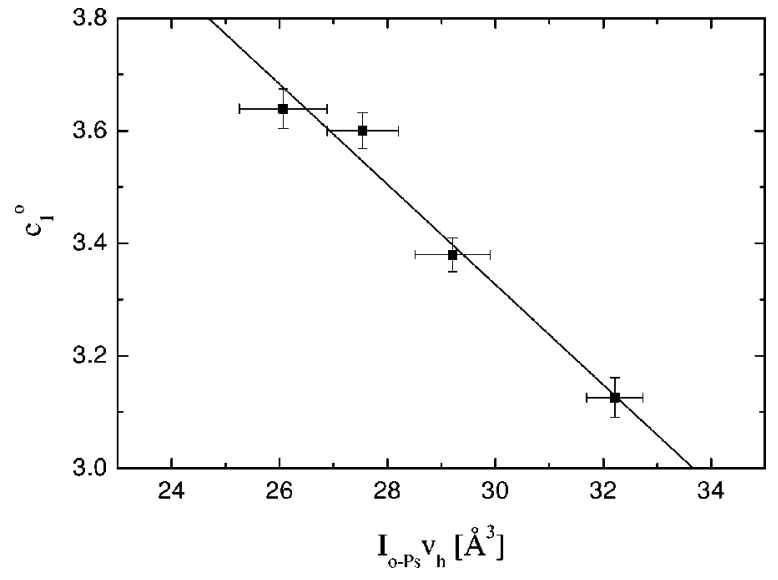

FIG. 4. Correlation between the WLF parameter $c_{1}^{0}$ and $I_{\mathrm{o}-\mathrm{Ps}} v_{h}$ from PALS measurements for SBR vulcanizates.

elastomers in which the free volume fraction and thermal expansion coefficient were evaluated. They concluded that a combination of both techniques could give more information about the behavior of the free volume in polymers and the dynamics associated with this free volume.

In order to use the information obtained using PALS measurements together with that from the dynamic mechanical tests, from Eqs. (4) and (7) it is easy to obtain

$$
c_{1}^{0}=\frac{B}{2.303 A I_{\mathrm{o}-\mathrm{Ps}} v_{h}} .
$$

When the parameter $c_{1}^{0}$, obtained by using the WLF relationship in the dynamic mechanical tests, is compared with the data from the positron tests for all the cured samples, a good correlation is obtained, as can be seen in Fig. 4. On the basis of this comparison it can be concluded that both techniques have apparently captured the same information. In other words, our results support the free volume theory.

\section{CONCLUSIONS}

In this work, an experimental investigation was undertaken to assess the effect of the time of cure on the changes of free volume size and fraction of free volume present during curing of styrene butadiene rubber. Measurements of positron annihilation lifetime spectroscopy were performed on uncured and partially cured samples. These data were complemented by a dynamic mechanical test performed on the vulcanized material.

The total free volume content, as measured by PALS, demonstrates that the free volume size significantly de-

TABLE III. Parameters of the WLF relationship at a reference temperature of $T_{0}=298 \mathrm{~K}$.

\begin{tabular}{lcccc}
\hline \hline Cure time (min) & $c_{1}^{0}$ & $c_{2}^{0}(\mathrm{deg})$ & $\alpha_{f} / B\left(10^{-4} \mathrm{deg}^{-1}\right)$ & $f_{0}$ \\
\hline 12.6 & $3.600 \pm 0.122$ & $107.7 \pm 3.6$ & 11.2 & 0.121 \\
15.1 & $3.636 \pm 0.075$ & $109.3 \pm 2.9$ & 10.9 & 0.119 \\
18.7 & $3.380 \pm 0.030$ & $105.3 \pm 2.2$ & 12.2 & 0.128 \\
31.2 & $3.126 \pm 0.035$ & $104.7 \pm 5.9$ & 13.3 & 0.139 \\
\hline \hline
\end{tabular}


creases at the onset of the reaction and slightly increases at higher levels of cure. From the present results, it can be concluded that the presence of cross links decreases the volume of the holes in the samples, restricting the thermal expansion of the chain.

The free volume fraction increases at high cure levels and the same tendency is observed in the results obtained with the WLF as well as with the PALS technique. This behavior can be associated with the creation of new cross links during curing and also with side reactions occurring during sulfur vulcanization.

Finally, when the parameter obtained by using the WLF relationship in a dynamic mechanical test is compared with PALS results, a good correlation between the experimental results is found, indicating that the two techniques give es- sentially the same information. These results suggest that positrons in SBR reflect the same basic mechanism as relaxation processes. So PALS measurements can be used to obtain information on dynamic processes in elastomeric materials.

\section{ACKNOWLEDGMENTS}

This work was supported by the University of Buenos Aires, Argentina (Investigation Project No. TY05), Consejo Nacional de Investigaciones Científicas y Técnicas (PIP/BID No. 4318/97), Comisión de Investigaciones Científicas de la Provincia de Buenos Aires, Secretaría de Ciencia y Técnica (UNCentro), Argentina, and Fundación Antorchas.
[1] J.D. Ferry, R.G. Mancke, E. Maekawa, Y. Oyanagi, and R.A. Dickie, J. Phys. Chem. 68, 3414 (1964).

[2] R. Payne, in Reinforcement of Elastomers, edited by G. Kraus (Interscience, New York, 1965), p. 64.

[3] A.J. Marzocca, C.L. Matteo, J.J. Gonzalez, and R.B. Raimondo, J. Phys. IV C8, 583 (1996).

[4] H.D. Heinze, K. Schmieder, G. Schnell, and K.A. Wolf, Kautsch. Gummi Kunstst. 14, 208 (1961).

[5] O. Krames, S. Hvidt, and J.D. Ferry, in Science and Technology of Rubber, edited by J.E. Mark, B. Erman, and F.R. Eirich (Academic Press, San Diego, 1994), p. 228.

[6] M. Mori and J.L. Koenig, Rubber Chem. Technol. 68, 551 (1995)

[7] N.G. McCrum, B.E. Read, and G. Williams, Anelastic and Dielectric Effects in Polymeric Solids (Dover, New York, 1991), p. 398.

[8] P. Mason, Polymer 5, 625 (1964).

[9] J.D. Ferry, in Viscoelastic Properties of Polymers (John Wiley \& Sons, New York, 1980).

[10] J.G. Victor and J.M. Torkelson, Macromolecules 20, 2241 (1987).

[11] R.B. Gregory, J. Appl. Phys. 70, 4665 (1991).

[12] Y.C. Jean, Microchem. J. 42, 72 (1990); Mater. Sci. Forum 59, 175-178 (1995).

[13] G. Dlubek, H.M. Fretwell, and M.A. Alam, Macromolecules 33, 87 (2000).
[14] O.E. Mogensen, Positron Annihilation in Chemistry (SpringerVerlag, Berlin, 1995).

[15] S.J.J. Tao, J. Chem. Phys. 56, 5499 (1972).

[16] M. Eldrup, D. Lightbody, and N. Sherwood, J. Chem. Phys. 63, 51 (1981).

[17] C. Wastlund and F.H.J. Maurer, Macromolecules 30, 5870 (1997).

[18] D.A. Hills, Heat Transfer and Vulcanization of Rubbers (Elsevier, London, 1971).

[19] P. Kirkegaard, N.J. Pedersen, and M. Eldrup, computer code PATFIT Ris $\phi-M-2740$, RNL, Roskilde, Denmark, 1989.

[20] J. Kansy, Nucl. Instrum. Methods Phys. Res. A 374, 235 (1996).

[21] T. Kanaya, T. Tsukushi, K. Kaji, J. Bartos, and J. Kristiak, Phys. Rev. E 60, 1906 (1999).

[22] A.K. Sircar, in Thermal Characterization of Polymeric Materials, edited by E.A. Turi (Academic Press, San Diego, 1997).

[23] R.A. Venditti, J.K. Gillham, Y.C. Jean, and Y. Lou, J. Coat. Technol. 67, 47 (1995).

[24] M. Akiba and S. Hashim, Prog. Polym. Sci. 22, 475 (1997).

[25] M.R. Krejsa and J.L. Koenig, Rubber Chem. Technol. 66, 376 (1995).

[26] R. Srithawatpong, Z.L. Peng, B.G. Olson, A.M. Jamieson, R. Simha, J.D. Mcgervey, T.R. Maier, A.F. Halasa, and H. Isida, J. Polym. Sci., Part B: Polym. Phys. 37, 2754 (1999).

[27] P. Bandzuch, J. Kristiak, O. Sausa, and J. Zrubcova, Phys. Rev. B 61, 8784 (2000). 